\title{
Long-term field application of sewage sludge increases the abundance of antibiotic resistance genes in soil
}

\author{
Qinglin Chen ${ }^{a}$, Xinli $\mathrm{An}^{\mathrm{a}}{ }^{\mathrm{a}}, \mathrm{Hu} \mathrm{Li}^{\mathrm{a}}$, Jianqiang $\mathrm{Su}^{\mathrm{a}}$, Yibing Ma ${ }^{\mathrm{b}}$, Yong-Guan $\mathrm{Zhu}{ }^{\mathrm{a}, \mathrm{c}, *}$ \\ a Key Lab of Urban Environment and Health, Institute of Urban Environment, Chinese Academy of Sciences, Xiamen 361021, China

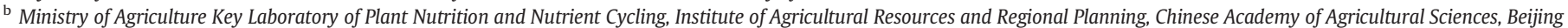 \\ 100081, China \\ c State Key Lab of Urban and Regional Ecology, Research Center for Eco-environmental Sciences, Chinese Academy of Sciences, Beijing 100085, China
}

\section{A R T I C L E I N F O}

\section{Article history:}

Received 7 February 2016

Received in revised form 21 March 2016

Accepted 21 March 2016

Available online 2 April 2016

\section{Keywords:}

Antibiotic resistance genes

Bacterial pathogens

Horizontal gene transfer

Network analysis

Sewage sludge

\begin{abstract}
A B S T R A C T
Sewage sludge and manure are common soil amendments in crop production; however, their impact on the abundance and diversity of the antibiotic resistome in soil remains elusive. In this study, by using highthroughput sequencing and high-throughput quantitative PCR, the patterns of bacterial community and antibiotic resistance genes (ARGs) in a long-term field experiment were investigated to gain insights into these impacts. A total of 130 unique ARGs and 5 mobile genetic elements (MGEs) were detected and the long-term application of sewage sludge and chicken manure significantly increased the abundance and diversity of ARGs in the soil. Genes conferring resistance to beta-lactams, tetracyclines, and multiple drugs were dominant in the samples. Sewage sludge or chicken manure applications caused significant enrichment of 108 unique ARGs and MGEs with a maximum enrichment of up to 3845 folds for mexF. The enrichment of MGEs suggested that the application of sewage sludge or manure may accelerate the dissemination of ARGs in soil through horizontal gene transfer (HGT). Based on the co-occurrence pattern of ARGs subtypes revealed by network analysis, aacC, oprD and $m p h A-02$, were proposed to be potential indicators for quantitative estimation of the co-occurring ARGs subtypes abundance by power functions. The application of sewage sludge and manure resulted in significant increase of bacterial diversity in soil, Proteobacteria, Acidobacteria, Actinobacteria and Chloroflexi were the dominant phyla $(>10 \%$ in each sample). Five bacterial phyla (Chloroflexi, Planctomycetes, Firmicutes, Gemmatimonadetes and Bacteroidetes) were found to be significantly correlated with the ARGs in soil. Mantel test and variation partitioning analysis (VPA) suggested that bacterial community shifts, rather than MGEs, is the major driver shaping the antibiotic resistome. Additionally, the co-occurrence pattern between ARGs and microbial taxa revealed by network analysis indicated that four bacterial families might be potential hosts of ARGs. These results may shed light on the mechanism underlining the effects of amendments of sewage sludge or manure on the occurrence and dissemination of ARGs in soil.
\end{abstract}

(c) 2016 Elsevier Ltd. All rights reserved.

\section{Introduction}

The persistence and spread of antibiotic resistance genes (ARGs) in the environment are promoted by anthropogenic activities such as sewage sludge applications in arable land (Threedeach et al., 2012). Municipal wastewater appears to be a significant reservoir of ARGs (Lachmayr et al., 2009), however, most ARGs cannot be effectively removed by wastewater treatment processes. ARGs have been detected at all stages of the municipal wastewater treatment process (Lachmayr et al., 2009; Munir et al., 2011), and the vast majority of ARGs are discharged from the municipal wastewater treatment process together

\footnotetext{
* Corresponding author at: Key Lab of Urban Environment and Health, Institute of Urban Environment, Chinese Academy of Sciences, No.1799 Jimei Road, Xiamen 361021, China.

E-mail address: ygzhu@iue.ac.cn (Y.-G. Zhu).
}

with sewage sludge (Bondarczuk et al., 2016). Land application is one of the key management approaches of sewage sludge from wastewater treatment plants (Kim and Aga, 2007). This practice is cost effective with added benefits from its residual nutrients (Candela et al., 2007), but it also represents a major pathway of ARGs spread onto farmlands (Chen and Zhang, 2013). Land application of sewage sludge may result in the spread of ARGs in soil and to underground water (Threedeach et al., 2012). More importantly, bacteria have been shown to readily share genetic information by HGT via mobile genetic elements (MGEs) including plasmids, transposons, and integrons (Zhu et al., 2013; Pruden et al., 2006), allowing the transfer of resistance genes from sewage sludge microorganisms to indigenous environmental bacteria (Gaze et al., 2011). Sewage sludge has been shown to be a hotspot for bacteria carrying ARGs and MGEs (Su et al., 2015) and its application may enhance the HGT of ARGs in soil, although the bacterial populations of sewage sludge and soil may be quite distinct (Hammesfahr et al., 
2008). Nevertheless, the fate of ARGs in arable soils following the longterm application of sewage sludge still remains poorly understood (Burch et al., 2014).

Animal manure represents a major source of antibiotics and ARGs in the environment (Wang et al., 2011; Binh et al., 2008, Binh et al., 2009). Manure disposal may transfer ARGs into the environment through a variety of pathways connected with common practice - land application (Joy et al., 2013; Wu et al., 2010). As a common practice, manure application introduces not only nutrients and organic matter for crop growth, but also antimicrobial agents into arable soil (Xiong et al., 2015; Cheng et al., 2013). Several studies have examined the effects of manure application on antibiotic resistance in soil from various perspectives, showing a stimulation effect of agricultural usage of manure containing antibiotics on the dissemination of antibiotic resistance in soil bacterial communities (Jechalke et al., 2013; Tang et al., 2015; Kyselková et al., 2015).

In general, for minimizing the effects of fluctuation of soil environmental conditions on microbial community, laboratory microcosm experiments have been commonly used in many studies (Chen et al., 2014; Chen et al., 2015). For instance, some studies have investigated the impacts of the application of sewage sludge on soil ARGs in a microcosm incubation experiment (Burch et al., 2014). However, due to the different conditions between laboratory and field experiments (Rahube et al., 2014), the results of previous studies may not completely reflect the impacts in field. In this study, we compared the impact of the application of sewage sludge with chicken manure (served as a positive control) and chemical fertilizer (with no obvious antibiotic-related properties) on soil resistome. To the best of our knowledge, there are only a few studies that systematically evaluate the fate and transport of ARGs in soil following the long-term application of sewage sludge. Therefore, by using Illumina sequencing and high throughput quantitative PCR (HT-qPCR) including 295 primer sets targeting almost all major classes of ARGs, the objectives of this study were to (1) evaluate the effects of long-term application of sewage sludge and manure on the abundance and diversity of ARGs, bacterial communities structure and potential bacterial pathogens in soil; (2) using network analysis to explore the indicator ARGs for the quick estimation of ARGs in environmental samples; (3) explore the co-occurrence patterns among ARGs subtypes, and between ARGs and microbial populations.

\section{Materials and methods}

\subsection{Sampling site and sample collection}

The field experiment was set up in a fluvo-aquic soil in the long-term experiment station of the Chinese Academy of Agricultural Sciences (CAAS), in Dezhou of Shandong Province, China ( $37^{\circ} 20^{\prime} \mathrm{N}, 116^{\circ} 38^{\prime} \mathrm{E}$ ). The experiment began in 2006, aimed at investigating the $\mathrm{N}$ and $\mathrm{P}$ input-output balances and soil $\mathrm{P}$ accumulation when sewage sludge and chicken manure were applied to a soil. Totally, eight treatments (i.e. $\mathrm{CK}, 0.5 \mathrm{~N}, 1 \mathrm{~N}, 0.5 \mathrm{SS}, 1 \mathrm{SS}, 2 \mathrm{SS}, 4 \mathrm{SS}, 1 \mathrm{CM}$ ) were carried out and the detailed information about the design of this field experiment is shown in Figure S1 and Table 1. The basic information about the soil, sewage sludge and chicken manure is listed in Table S1. We collected 24 samples (surface soil $0-15 \mathrm{~cm}$ ) covering all treatments with triplicate in August 2015 (about ten months after the application of sewage sludge and chicken manure). The soils were immediately frozen on dry ice, transported to laboratory within $24 \mathrm{~h}$ and stored at $-80^{\circ} \mathrm{C}$ before analysis.

\subsection{DNA extraction}

DNA was extracted from $0.5 \mathrm{~g}$ frozen samples using a FastDNA® Spin Kit for soil (MP Biomedical, Santa Ana, California, USA) according to the manufacturer's instructions. The quality of the DNA was checked by spectrophotometric analysis using NanoDrop ND-1000 (Nanodrop,
Table 1

The application rates of sewage sludge, chicken manure and chemical fertilizers in different treatments.

\begin{tabular}{|c|c|c|c|c|c|}
\hline Treatment & $\begin{array}{l}\text { Sewage } \\
\text { sludge }^{a}\end{array}$ & $\begin{array}{l}\text { Chicken } \\
\text { manure }\end{array}$ & Urea & Superphosphate & $\begin{array}{l}\text { Potassium } \\
\text { sulphate }\end{array}$ \\
\hline & $\mathrm{t} \cdot \mathrm{hm}^{-2}$ & $\mathrm{t} \cdot \mathrm{hm}^{-2}$ & $\mathrm{~kg} \cdot \mathrm{hm}^{-2}$ & $\mathrm{~kg} \cdot \mathrm{hm}^{-2}$ & $\mathrm{~kg} \cdot \mathrm{hm}^{-2}$ \\
\hline CK & 0 & 0 & 0 & 600 & 240 \\
\hline $0.5 \mathrm{~N}$ & 0 & 0 & 65.25 & 600 & 240 \\
\hline $1 \mathrm{~N}$ & 0 & 0 & 130.50 & 600 & 240 \\
\hline $0.5 S S$ & 4.5 & 0 & 65.25 & 600 & 240 \\
\hline $1 \mathrm{SS}$ & 9 & 0 & 65.25 & 600 & 240 \\
\hline $2 S S$ & 18 & 0 & 65.25 & 600 & 240 \\
\hline $4 S S$ & 36 & 0 & 65.25 & 600 & 240 \\
\hline $1 \mathrm{CM}$ & 0 & 10 & 65.25 & 600 & 240 \\
\hline
\end{tabular}

a Before wheat sowing every year (mid of October), the air-dried sewage sludge was applied as the basic fertilizer incorporated into the soil.

USA) and the concentration of DNA was determined using QuantiFluor ${ }^{\circledR}$ dsDNA system (Promega, Madison, Wisconsin, USA) using fluorometric analysis with a microplate reader (Spectramax M5, USA). Soil DNA was stored at $-20^{\circ} \mathrm{C}$ until use.

\subsection{High-throughput quantitative PCR}

High-throughput qPCR reactions were performed using the Wafergen SmartChip Real-time PCR system. This system can be used for large scale gene expression studies, which can process 5184 nanowell reactions per run (Wang et al., 2014). A total of 296 primer sets were used, including 295 primer sets targeting almost all major classes of ARGs and MGEs and 1 16S rRNA gene (Su et al., 2015). After the initial enzyme activation at $95{ }^{\circ} \mathrm{C}$ for $10 \mathrm{~min}, 40$ cycles of the following program were used for amplification: denaturation at $95^{\circ} \mathrm{C}$ for $30 \mathrm{~s}$, annealing at $60^{\circ} \mathrm{C}$ for $30 \mathrm{~s}$. The melting process was automatically generated by Wafergen software. After that, results were analyzed with SmartChip qPCR Software excluding the wells with multiple melting peaks or amplification efficiency beyond the range (90\%-110\%) and then screened with conditions that (1) a threshold cycle $\left(\mathrm{C}_{\mathrm{T}}\right)$ must be $<31$ and (2) positive samples should have three replicates simultaneously. Relative copy number was calculated referring to a previous study (Eq.1) (Ou-yang et al., 2015). Besides, a comparative $C_{\mathrm{T}}$ method was used to calculate the ARGs' fold change (FC value) of amended samples compared to the control (Eq.2) (Schmittgen and Livak, 2008). The detection limit $C_{T}$ (31) was taken as a replacement for the genes with no amplification.

Gene copy number $=10^{\left(31-C_{\mathrm{T}}\right) /(10 / 3)}$

$\Delta \mathrm{C}_{\mathrm{T}}=\mathrm{C}_{\mathrm{T}(\mathrm{ARG})}-\mathrm{C}_{\mathrm{T}(16 \mathrm{~S})}$

$\Delta \Delta \mathrm{C}_{\mathrm{T}}=\mathrm{C}_{\mathrm{T}(\text { Target })}-\Delta \mathrm{C}_{\mathrm{T}(\text { Ref })}$

$\mathrm{FC}=2^{\left(-\Delta \Delta \mathrm{C}_{\mathrm{T}}\right)}$

Where $C_{T}$ is the threshold cycle, ARG is one of the 295 antibiotic resistance gene assays, $16 \mathrm{~S}$ is the $16 \mathrm{~S}$ rRNA gene assay, Target is the amended sample, Ref is the control sample.

\subsection{Illumina sequencing and analyses of $16 S$ rRNA gene}

The V4-V5 region of bacterial 16S rRNA was selected for amplification with primers F515: GTGCCAGCMGCCGCGG and R907: CCGTCAAT TCMTTTRAGTTT. To pool all samples for one run of Illumina sequencing, the reverse primers were designed with 24 unique barcodes. After the initial enzyme activation at $95^{\circ} \mathrm{C}$ for $5 \mathrm{~min}, 35$ cycles of the following program were used for amplification: $95^{\circ} \mathrm{C}$ for $30 \mathrm{~s}, 58^{\circ} \mathrm{C}$ for $30 \mathrm{~s}$ and $72{ }^{\circ} \mathrm{C}$ for $30 \mathrm{~s}$. Then, the amplification products were purified and submitted to Illumina Hiseq2000 platform (Novogene, Beijing, China) for sequencing. QIIME quality filters filtered the raw reads. The generated 
high quality sequences were processed and analyzed using QIIME ( $\mathrm{Su}$ et al., 2015). The default method was used for sequence processing quality control and OTU picking. OTU was defined at the $97 \%$ similarity level using UCLUST clustering (Edgar, 2010). Alpha diversity was described for each sample using the metrics observed species (i.e. OTUs), Chao1, Shannon index and PD Whole tree, and rarefaction curves were generated to compare the level of bacterial OTU diversity. Nonmetric multidimensional scaling (nMDS) analysis was performed to evaluate the overall profiles of microbial community among different treatments.

\subsection{Bacterial pathogens list}

Based on the taxonomic list derived from the virulence factors database (http://www.mgc.ac.cn/VFs/) and some references (Fang et al., 2015; Li et al., 2015a; Ye and Zhang, 2011), the bacterial pathogens database including 557 pathogenic species (Table S2) was constructed. All the 16S rRNA gene sequences of the selected bacterial pathogens are publicly available from the NCBI GenBank (http:// www.ncbi.nlm.nih.gov/). The 16S rRNA gene sequences from each sample were blasted against the bacterial pathogens $16 \mathrm{~S}$ database with an E-value $<1 \times 10^{-10}$. The blast hit outputs were further filtered to annotate the bacterial pathogens using the strict criteria of sequence identity $>99 \%$.

\subsection{Statistical analysis}

Averages, standard deviations, and fold change values of all data were determined using Microsoft Excel 2007. Figures were generated using SigmaPlot 11.0. Statistical analyses were performed using SPSS Software (PASW Statistics 20.0). All statistical tests were considered significant at $P<0.05$. Heat maps and variation partitioning analysis (VPA) for determination of the contributions of bacterial communities and MGEs to the variations of persistent ARGs were conducted in $\mathrm{R}$ with the package "pheatmap", "vegan" and "MASS". Principal coordinate analysis (PCOA) was performed to evaluate the overall pattern of ARGs and bacterial pathogens among the samples based on the Bray-Curtis distance. Correlation between bacterial community and ARGs was examined by Mantel test and redundancy analysis (RDA). Bacterial phyla were considered as the environmental factors affecting the ARGs variation in RDA. PCoA and RDA were performed using Canoco version 5.0 software. A correlation matrix by calculating all possible pair-wise Spearman's rank correlations between the ARGs subtypes was constructed to visualize the correlations in the network interface. A correlation between two items was considered statistically robust if the Spearman's correlation coefficient $\left(R^{2}\right)$ was $>0.8$ and the $P<0.01$ (Li et al., 2015b). The Cytoscape 3.3.0 software was used to visualize the network graphs using the circular layout algorithm (Halary et al., 2010; Yin et al., 2015).

\section{Results}

\subsection{Abundance and diversity of antibiotic resistance genes}

The application of sewage sludge and chicken manure changed the ARG diversity and detection frequency. A total of 130 unique ARGs and 5 MGEs (including class 1 integrase gene) were detected among all soil samples. ARGs were classed into 9 types (i.e. aminoglycoside, beta-lactams, chloramphenicol, Macrolide-Lincosamide-Streptogramin $B$ resistance genes (MLSB), multiple drugs, sulfonamide, tetracyclines, vancomycin and Others) based on the antibiotic classes they confer resistance to. The detection frequency ranged from 42 to 100 , with the highest detection frequency in the treatment 4SS (Figure S2). The detection frequency in soil fertilized with chemical fertilizer only was similar to the control plot, which was significantly lower than in sewage sludge and chicken manure- amended soils (except for 0.5SS). To minimize the variance caused by different extraction yields and differences of the background bacterial abundances, ARGs was normalized by $16 \mathrm{~S}$ rRNA (ARGs copies/cell). In the control soil (CK) the abundance of ARGs was 0.012 copies per cell. The application of sewage sludge (except for 0.5SS) and chicken manure increased the ARGs abundance with the highest abundance of ARGs in the treatment of 1CM. In addition, we observed a positive dose-effect in the treatments of sewage sludge. Additionally, the application of chemical fertilizer (1N) also caused a slight increase in ARG abundance (Figure S3).

Resistance genes to multiple drugs, MLSB, beta-lactams, tetracyclines and aminoglycoside were the most common types of ARGs in soils while chloramphenicol and sulfonamide were lower than other types of ARGs (Fig. 1). The application of sewage sludge at a high dose (4SS) and chicken manure caused a significant increase in the abundance of multiple drugs resistance genes and MGEs (Figure S4). In addition, the application of chicken manure increased the abundance of tetracycline resistance genes markedly. The application of chemical fertilizer and sewage sludge at low dose (0.5SS) caused a significant increase in aminoglycoside resistance genes. Based on the Bray-Curtis distance, PCoA analysis showed that CK clustered together and was separated from the other samples, indicating that the overall patterns of ARGs were altered by soil amendment (including $0.5 \mathrm{~N}$ and $1 \mathrm{~N}$ ). The first two PCs explained a total of $68.20 \%$ variance of ARGs, with PC1 explaining $46.52 \%$ and PC2 explaining $16 \%$ of the variance (Fig. $2 \mathrm{~A}$ ). The Shannon-Wiener and Inverse Simpson index (Table S3) indicated that the application of chemical fertilizer, sewage sludge and chicken manure increased the ARGs diversity, except for the treatment of 0.5SS.

\subsection{Enrichment of antibiotic resistance genes}

Among all the detected 135 unique ARGs (including MGEs), a total of 108 unique ARGs were enriched in soil. Twenty three to 66 unique ARGs were enriched among different treatments and 66 unique ARGs were enriched in the treatment of $1 \mathrm{CM}$. The heat map of the fold change (FC) value comparing different treatments versus the control soil was shown in Fig. 3. From the heat map, we observed that different types of ARGs had various extent of enrichment. Most of them were enriched while some ARGs were not enriched and some even decreased (marked with $A$ and $B$ in the heat map). The maximum enrichment of a unique ARG was up to 3845 fold (mexF) in the treatment of $1 \mathrm{CM}$. Other unique ARGs such as orpJ, floR, aadE, pikR2, ermB and ermD were enriched over 100 fold in treatments of sewage sludge or chicken manure.

\subsection{Co-occurrence patterns among ARGs subtypes}

The co-occurrence patterns among ARGs subtypes were explored using network analysis. Fig. 4 consists of 44 nodes (ARGs subtypes) and 118 edges with an average degree or node connectivity of 5.364. The average network distance between all pairs of nodes (average path length) was 1.898 edges with a network diameter of 5 edges. The clustering coefficient was 0.756 . Based on the modularity, the entire network could be parsed into five major modules, with 37 of 44 total vertices occupied by the three largest modules: Module I, III and IV. The most densely connected node in each module was defined as the 'hub'.

The 'aacC', 'oprD' and 'mphA-02' gene class of aminoglycoside, multiple drugs, and MLSB resistance genes were the hub of Module I, III and IV, respectively. The hubs and related co-occurring ARGs in each module might be harbored in some specific microbial taxa that are shared in the environment. Thus, we could use these hubs as the ARGs indicators to qualitatively and quantitatively analyze the corresponding co-occurring ARGs. Briefly, these three hubs could be used as representatives of 27 unique ARGs. In the Supplementary information we have tested the feasibility of using these 'hubs' as representative ARGs (Table S4, Table S5, Figure S5). The results indicated that the abundance of 'hubs' and the co-occurring ARGs followed a 


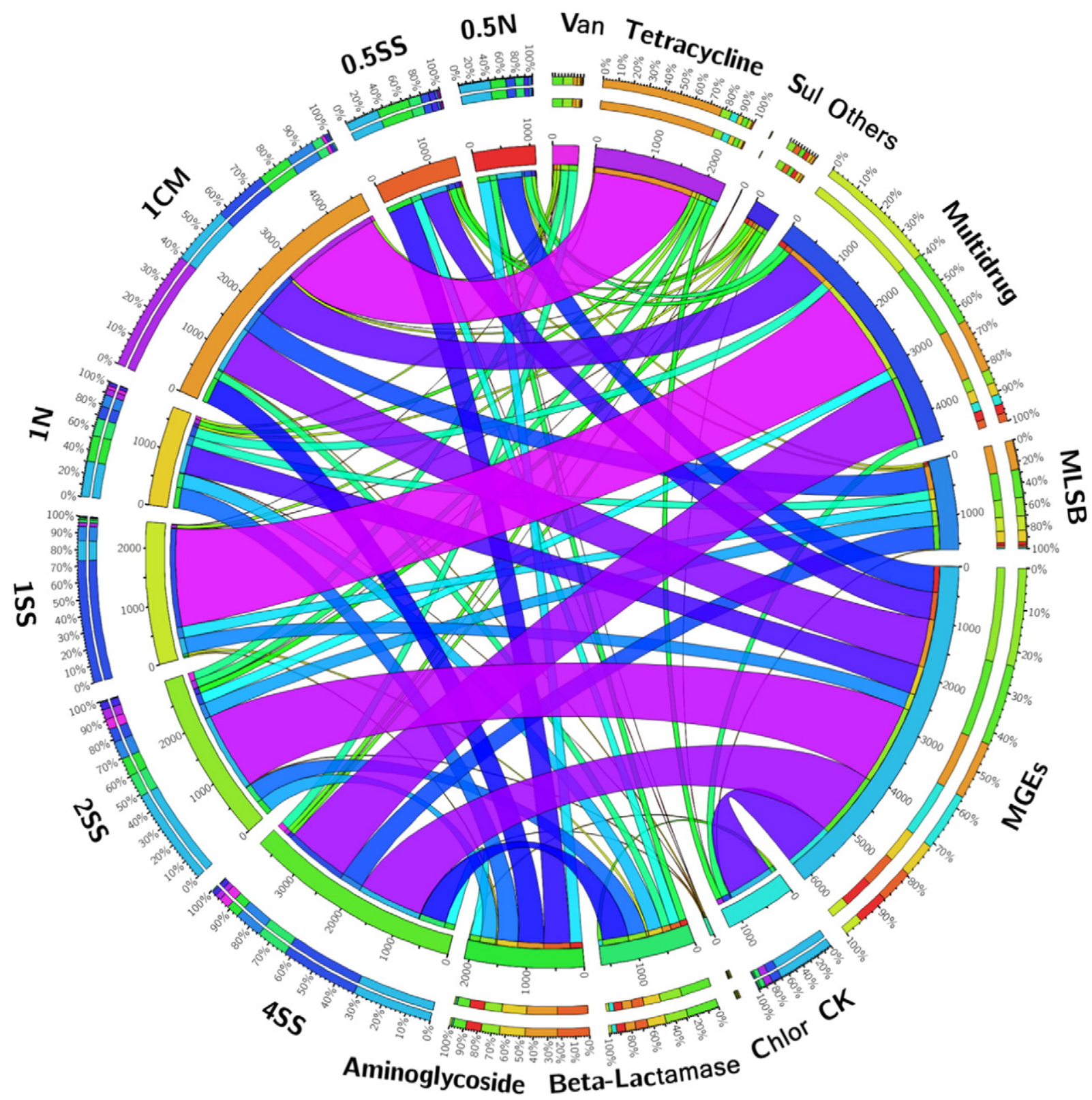

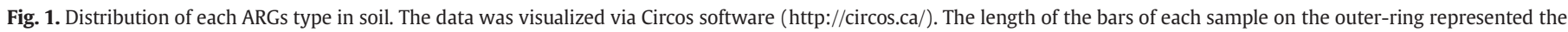
percentage of ARGs in each sample. MLSB: Macrolide-Lincosamide-Streptogramin B resistance genes, Van: Vancomycin, Sul: Sulfonamide, Chlor: Chloramphenicol.

power function with $R^{2}$ values ranging from 0.86 to 0.95 . We compared the predicted and detected values, the relative error for ' $a a c C$ ' gene, 'oprD' gene and 'mphA-02' gene as indicator to estimate the related cooccurring ARGs ranged from 0 to $79.11 \%, 0.34 \%$ to $45.04 \%$ and 0 to $24.39 \%$ (absolute value), respectively.

\subsection{Characterization of bacterial communities}

A total of 1,850,703 high quality sequences were obtained from all 24 samples with sequences per sample ranging from 60,918 to 94,152. These sequences were clustered into 58,217 OTUs at the $3 \%$ dissimilarity level, with an average of 11,851 OTUs per sample. The heat map of major phyla ( $>1 \%$ in any sample) based on phylogenetic classification showed that Proteobacteria was the dominant phylum with percentages ranging from $27.4 \%$ to $33.0 \%$ in all samples (Figure S6). As the second most abundant phylum in soil, the abundance of Acidobacteria decreased in treatments of 4SS and 1CM. In addition, the application of sewage sludge (especially at high doses) and chicken manure increased the abundance of Actinobacteria, while decreasing the abundance of Chloroflexi. At the class level, the differences in bacterial communities became more divergent. For instance, Alphaproteobacteria was most abundant in samples of $1 \mathrm{CM}, 1 \mathrm{SS}, 0.5 \mathrm{~N}$ and $\mathrm{CK}$, while in samples of 4SS the most abundant class shifted to Actinobacteria, and Acidobacteria-6 was dominant in the samples of 0.5SS, 2SS and $1 \mathrm{~N}$ (Figure S7). Furthermore, rarefaction curves of OTUs at the sequencing depth of 24,780 showed that bacterial alpha-diversity increased after the application of sewage sludge and chicken manure, which is confirmed by the evaluation of Chao1 estimator, phylogenetic diversity metrics (PD whole tree), observed species and Shannon index (Figure S8). Non-metric multidimensional scaling (nMDS) analysis (stress $=0.05$ ) revealed that the overall patterns of bacterial community were altered by soil amendments (Figure S9). RDA analysis indicated that the most dominant phylum Proteobacteria was positively correlated with soil total nitrogen (TN), total carbon (TC) and $\mathrm{pH}$, while negatively correlated with ratio of total carbon to nitrogen $(\mathrm{C} / \mathrm{N})$ (Figure S10). 

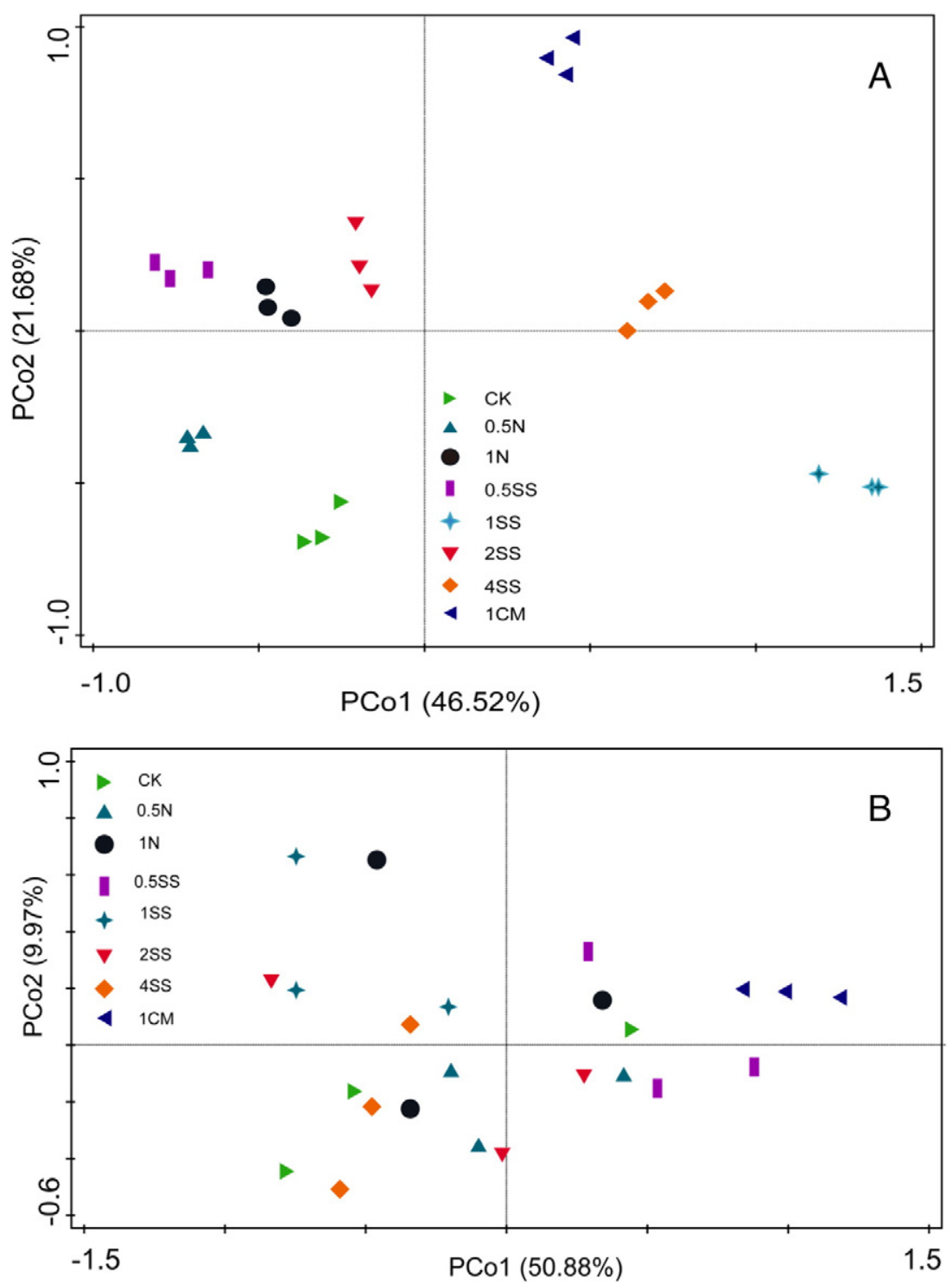

Fig. 2. Principal coordinate analysis (PCoA) based on the Bray-Curtis distance showing the overall distribution pattern of ARGs (A) and bacterial pathogens (B) in soil.

\subsection{Co-occurrence patterns between ARGs and microbial taxa}

Co-correlation networks are well suited to detect general patterns in highly populated taxonomic groups. Thus in the present study, the cooccurrence patterns between ARGs subtypes and microbial taxa (family-level) were also investigated using network analysis approach (Fig. 5). We hypothesized that the nonrandom co-occurrence patterns between ARGs and microbial taxa could indicate the possible host information of ARGs if the ARGs and the co-existed microbial taxa possessed a strong and significantly positive correlation (Spearman's $R^{2}>0.8, P<0.01$ ). As shown in Fig. 5, four bacterial families were thought to be the possible ARGs hosts based on the co-occurrence results. For instance, Rhodospirillaceae was the host of a beta-lactam resistance gene (blaCTX-M-02) and a multiple drugs resistance gene (mtrD-02). Syntrophobacteraceae was found to be the host of ARGs subtypes of tnpA-04 and ermT-01, whereas Gaiellaceae only carried tnpA-02. Compared with above families, Solirubrobacteraceae carried more diverse ARGs, including vancomycin resistance genes (vanHB, vanB-01), a multiple drugs resistance gene (acrA-05), beta-lactams resistance gene (ampC-04), and a MLSB resistance gene (mphA-02).

\subsection{Occurrence and abundance of bacterial pathogens}

In total, 34 bacterial pathogenic species were detected in all samples. Bacillus thuringiensis, Mycobacterium smegmatis and Bacillus megaterium were the dominant bacterial pathogens followed by Mycobacterium sp., Bacillus licheniformis, Mycobacterium gilvum, Pseudomonas aeruginosa and Pseudomonas fluorescens (Figure S11). Bacillus was the most common bacterial pathogen observed in this soil, however, most Bacillus are harmless saprophytes except for two species, which are considered medically significant, Bacillus anthracis and Bacillus cereus. In this study B. anthracis and B. cereus were not detected in any sample. While two other important human pathogens, Mycobacterium tuberculosis and Pseudomonas aeruginosa, were detected in all samples. The PCoA analysis showed that the treatment of $1 \mathrm{CM}$ was clustered together and separated from the other samples, which indicated that the application of chicken manure shifted the pattern of soil bacterial pathogens. However, the different treatments of sewage sludge did not cluster together or separated from the other samples, indicating that application of sewage sludge did not significantly shift the overall pattern of bacterial pathogens in soils (Fig. 2B). 


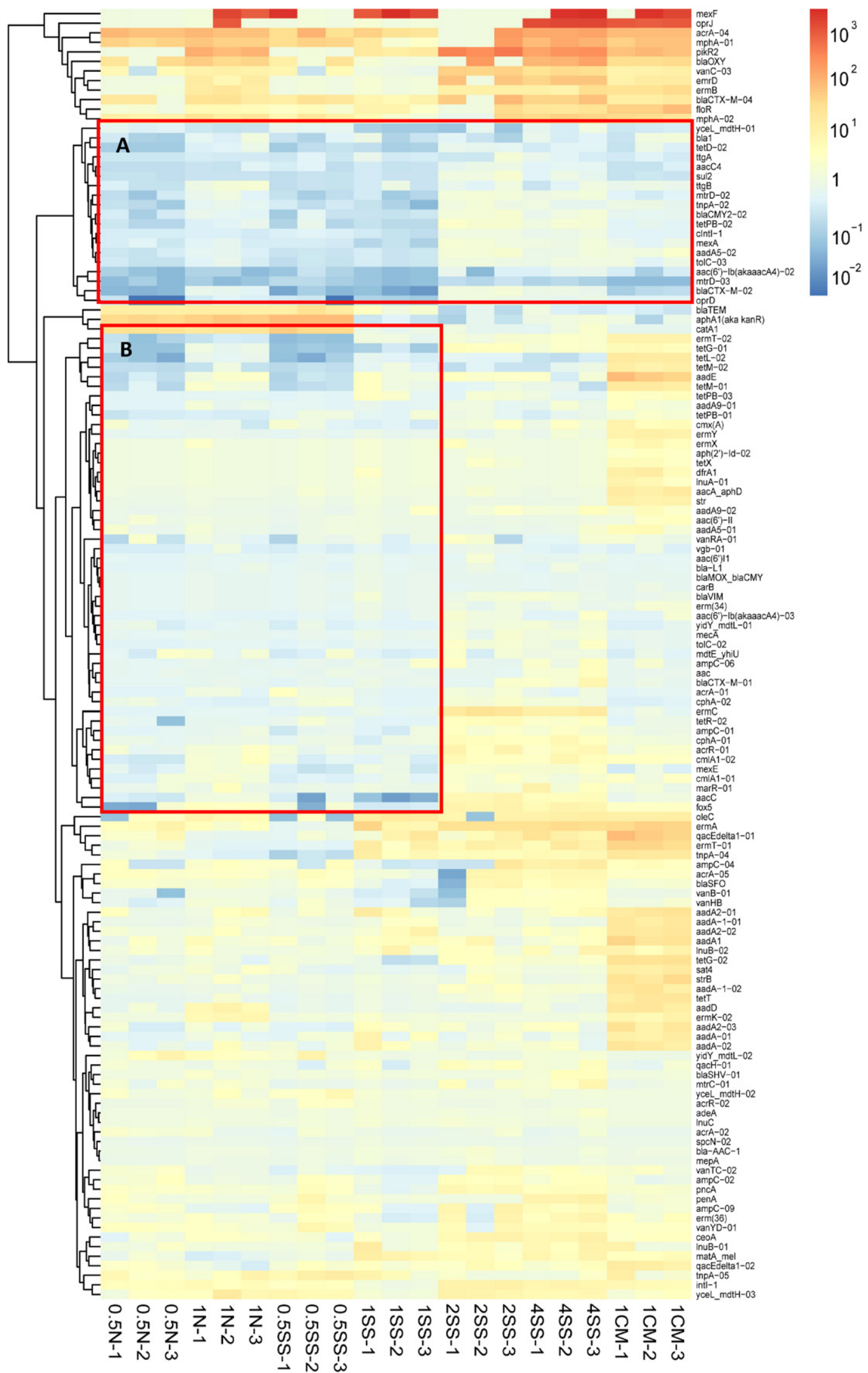

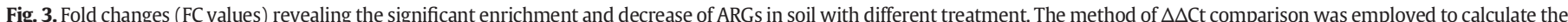

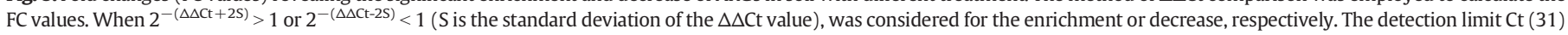
was taken as a replacement for the genes with no amplification. 


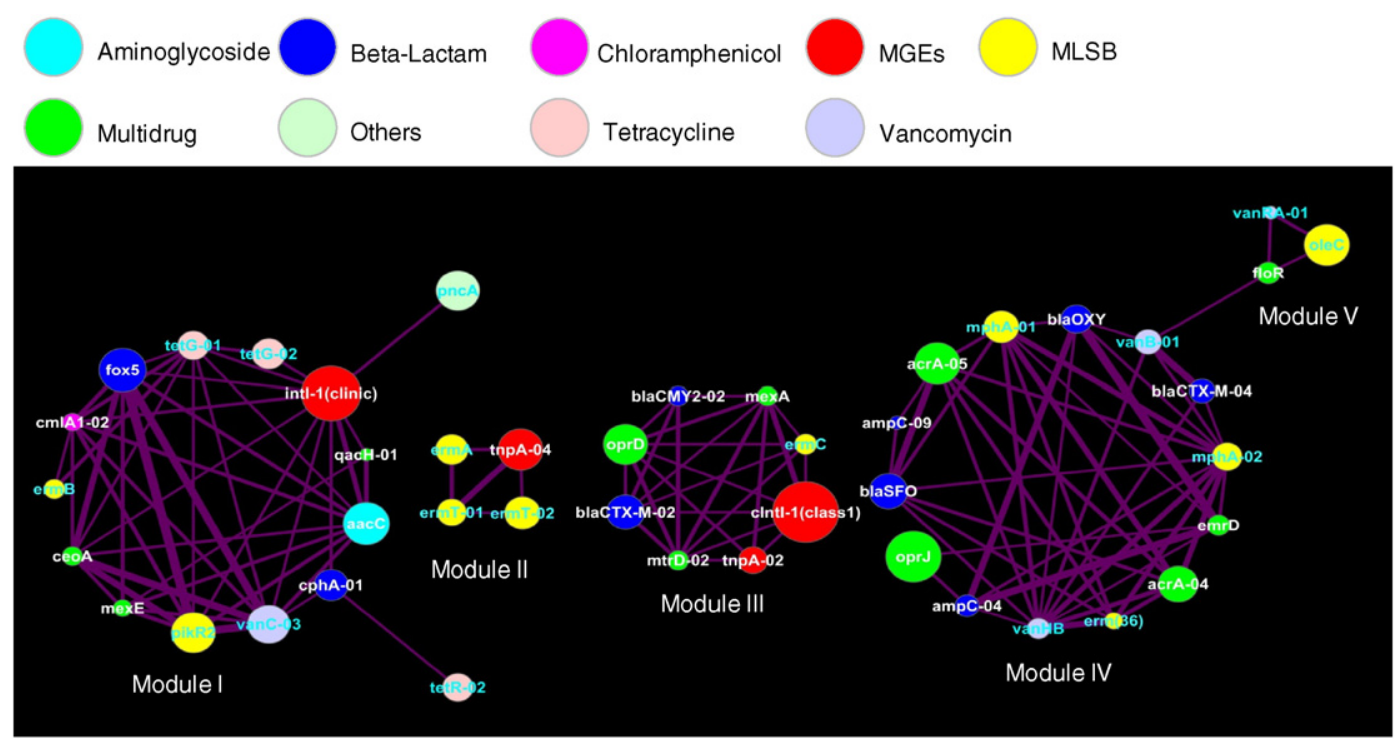

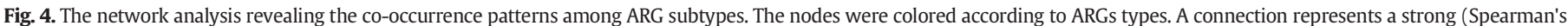

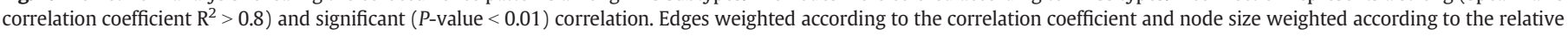
abundance of ARGs.

\subsection{Correlation analysis}

The abundance of total ARGs was positively correlated with MGEs $(P<0.01)$ (Table S6). We also observed that MGEs were positively correlated with genes conferring resistance to chloramphenicol $(P<0.01)$, beta-lactams $(P<0.01)$ MLSB $(P<0.05)$, Others $(P<0.01)$, sulfonamide $(P<0.01)$ and vancomycin $(P<0.01)$. More information about the correlation was provided in Table S6. RDA and VPA analysis (Fig. 6) showed that a total of $82.0 \%$ variance of ARGs could be explained by selected variables. TN and TC exhibited a significant positive relationship with the abundance of ARGs in soil, while the $\mathrm{C} / \mathrm{N}$ on the other hand was significantly negatively correlated with the abundance of ARGs.
Additionally, soil pH exhibited no significant correlation with ARGs. Five phyla (Chloroflexi, Planctomycetes, Firmicutes, Gemmatimonadetes and Bacteroidetes) were found significantly correlated with the ARGs in soil $(P<0.05)$. Chloroflexi, and Planctomycetes were positively correlated with the first axis (explaining $68.3 \%$ of total variance) and treatment of 1CM. Firmicutes and Gemmatimonadetes were positively correlated with ARGs abundance in the treatment of 4SS. However, the phylum Bacteroidetes was negatively correlated with the first axis but positively correlated with the second axis (explaining $14.5 \%$ of total variance) in treatments of $\mathrm{CK}, 0.5 \mathrm{~N}$ and $1 \mathrm{~N}$. VPA analysis indicated that bacterial community shift contributed to $39.8 \%$ of the ARGs variation, and the interaction between environmental factors and bacterial community explained

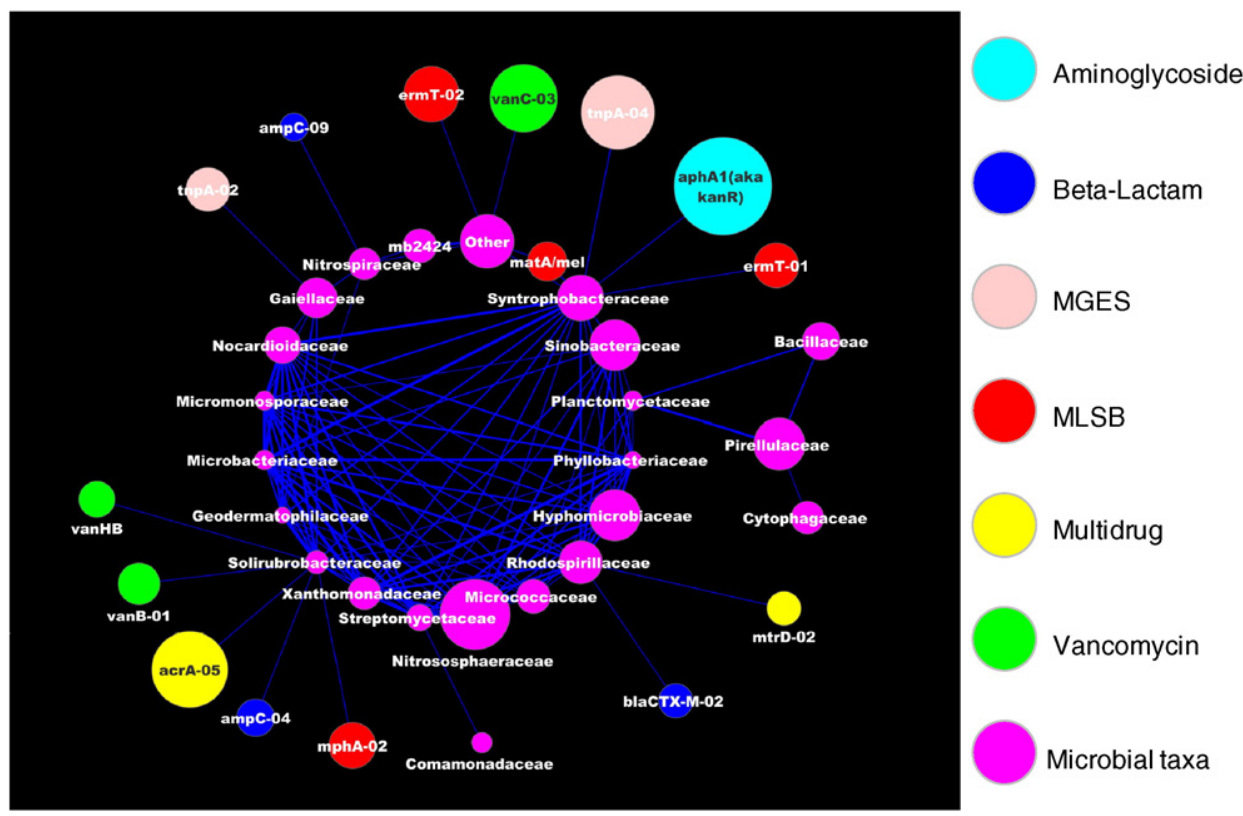

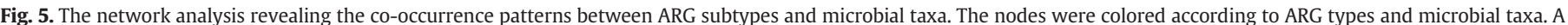

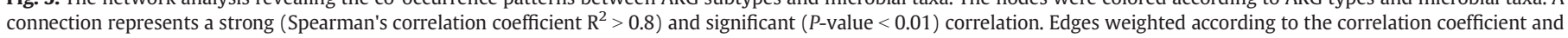
node size weighted according to the relative abundance of ARGs/microbial taxa. 


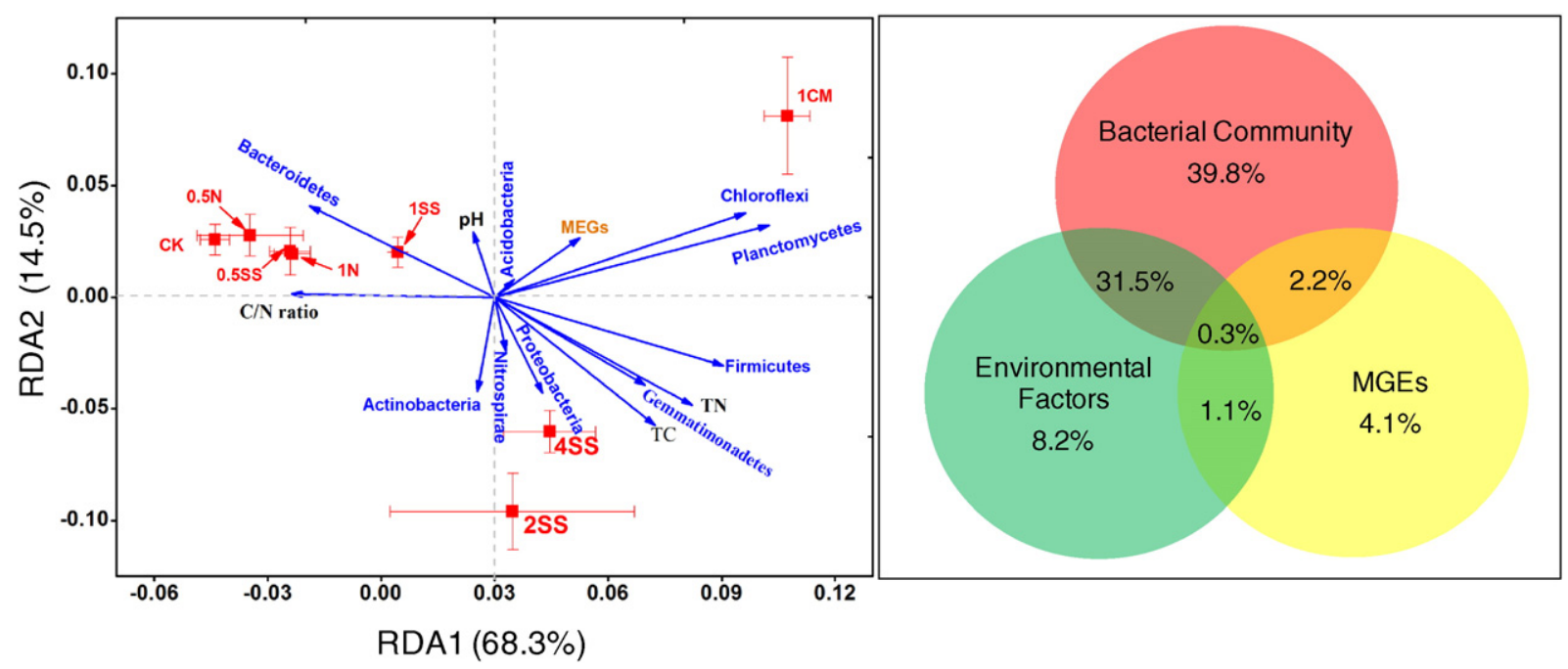

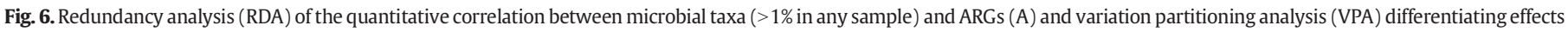
of bacterial community, environmental factors and mobile genetic elements (MGEs) on the resistome alteration (B).

31.5\% variation in soil, which was much higher than environmental factors (explained $8.2 \%$ variation) and MGEs (explained $4.1 \%$ variation). In addition, Mantel test showed that bacterial community structures were significantly correlated with ARGs profiles based on Bray-Curtis distance $(\mathrm{r}=0.41, P=0.0045)$.

\section{Discussion}

\subsection{Responses of ARGs abundance and composition}

This study demonstrated that the application of sewage sludge and chicken manure increased the abundance of ARGs in soil (Fig. 1, Figure S3), and antibiotic deactivation and efflux pump were the two dominant resistance mechanisms (Figure S12). The results were in agreement with previous studies (Rahube et al., 2014; Burch et al., 2014). The application of sewage sludge and chicken manure changed the ratio of deactivation to efflux pump genes, indicating that the soil ARGs composition was shifted. The PCoA analysis and the diversity index value also indicated that the ARGs composition was influenced by sewage sludge and chicken manure (Fig. 2, table S3). Previous study found that ARGs with manure application accounted for approximately $70 \%$ of the total ARGs in soil, which suggested that manure amendment increased the diversity of ARGs in soil (Su et al., 2014). In addition, we observed a positive dose-effect on the abundance of ARGs in treatments of sewage sludge, and the lower dose (0.5SS) caused no significant increase in the abundance of ARGs. Thus, we suggest that $0.5 \mathrm{SS}$ may be a safe dose for land application.

Intriguingly, compared with the $\mathrm{CK}$, the application of chemical fertilizer increased the ARGs abundance and diversity, although to a much lesser extent. This phenomenon may be due, at least partly, to potential changes in soil microbial composition including the native soil antibiotic-resistant bacteria (ARB) after the application of chemical fertilizer (Forsberg et al., 2014). Although chemical fertilizer had some effects on soil ARGs, these effects, not surprisingly, are weaker when compared with sewage sludge or chicken manure, considering that manure is rich in ARGs and ARBs (Yang et al., 2014, Jiang et al., 2002; Ghosh and LaPara, 2007) and most ARGs are discharged from the municipal wastewater treatment process in the form of sewage sludge (Munir et al., 2011). However, land application of animal manure or sewage sludge does not universally increase ARGs in soils (Zhou et al., 2010) and this increase is not maintained long-term (Fahrenfeld et al., 2014). For instance, manure from three dairy farms did not increase cultivable chlortetracycline-resistant bacteria when land-applied to soil (Ghosh and LaPara, 2007). Also in a microcosm study, the relative abundance of tetAP, tet $X$, and tet $O$ genes decreased over time, and fell below the initial levels of the control soils during the 60d experimental periods (Heuer et al., 2008). These discoveries were not consistent with our results; multiple factors could result in these inconsistent results. On the one hand, differences between culture-dependent and cultureindependent methods and differences between a microcosm study and a long-term field experiment may take account for this inconsistence. For instance, cultivation-based methods, capture only the culturable fraction of the total bacterial population ( $0.1-10 \%$ of microorganisms occurring in soil) capable of growing under laboratory conditions (Littlefield-Wyer et al., 2008). In the present study, we using HT-qPCR including 295 primer sets targeting almost all major classes of ARGs, which can provide more comprehensive pattern of ARGs than the common qPCR. In addition, we cannot rule out the effect of different soil and waiting period between the last treatment and sampling time from our study (in the present study the waiting period is about ten months). On the other hand, the fate and transport of ARGs could be affected by anthropogenic activities, such as crop planting and harvest.

\subsection{Horizontal transfer of antibiotic resistance genes}

We found that MGEs were significantly positively correlated with total ARGs in soil indicating MGEs may play a role in the dissemination of ARGs. HGT could potentially enhance the accumulation and persistence of ARGs in manure/sludge-amended soil via MGEs (Gaze et al., 2011; Smalla et al., 2000). It has been demonstrated that MGEs, especially integrons can be used by bacteria to stockpile and express different exogenous resistance genes and are considered to play a central role in the worldwide problem of antibiotic resistance (Gaze et al., 2011; Gillings et al., 2008; Partridge et al., 2009). Our results indicated that MGEs had significantly positive correlation with resistance genes to beta-lactams, MLSB, sulfonamide and vancomycin, while no significant correlation with resistance genes to tetracyclines. Thus we inferred that the HGT occurrence of resistance genes may depend, at least partly, on its types. The limited distribution of some genes is possibly associated with their limited host range, such as tetE, since it is associated with large plasmids, which are neither mobile nor conjugative (Ng et al., 2011). This may reduce its opportunities to transfer to other species (Chopra and Roberts, 2001). 


\subsection{Linkages between bacterial community and antibiotic resistome}

The composition of microbial communities can affect the occurrence and abundance of ARGs, but the information on the impact of microbial community composition on ARGs profiles in environmental samples was scarce. ARGs profiles were positively correlated to the relative abundance of Actinobacteria, whose abundance was higher in treatments of sewage sludge and chicken manure (Fig. 3 and Fig. 5). As a well-known group of antibiotic producing bacteria, Actinobacteria were often detected with multiple drugs resistance which could contribute to the elevated abundance and enrichment of ARGs ( $\mathrm{Su}$ et al., 2015). An important finding from this work is that bacterial community shifts play a dominant role in shaping the antibiotic resistome in soil. Although, HGT via MGEs is often regarded as the underlying mechanism responsible for the resistome formation and spread under different ecological niches (Jia et al., 2015). In this study MGEs explained only $4.1 \%$ variation of ARGs. It has been suggested that it is the phylogeny, rather than HGT as the primary determinant of soil resistome content (Forsberg et al., 2012; Forsberg et al., 2014). Therefore, as bacterial diversity changes across soils, so do their associated ARGs, resulting in resistome that may respond to anthropogenic modulations that even do not possess obvious antibiotic-related properties (such as chemical fertilizer). The similar results were found in a recent study on drinking water system indicating that the major driver of antibiotic resistome alternation was bacterial community shift rather than MGEs reproduction or transference (Jia et al., 2015). In addition, our previous work also found that MGEs contributed only $2.6 \%$ of the resistome variation during sewage sludge composting ( $\mathrm{Su}$ et al., 2015). Nevertheless, the positive correlation between MGEs and total ARGs was observed in this study suggesting that the potential of HGT in dissemination and spreading of ARGs should not be overlooked. Therefore it is necessary to learn more about ARGs profiles as well as microbial community composition during the long-term land application of sewage sludge, in order to further delineate the relationship between ARGs and bacterial communities and provide more information on the control of ARGs.

\subsection{Network analysis}

We explored the ARGs - ARGs and ARGs - microbial taxa associations using network analysis, and demonstrated it is an effective approach for exploring ARGs indicators and the possible ARGs hosts. Based on ARGs indicators, we could qualitatively and quantitatively analyze the corresponding co-occurring ARGs. Briefly, to indicate the abundance of the co-occurring ARGs in samples, a simple and quick qPCR assay of the ARGs indicator as the target genes could be used. This will substantially save the detection time and labor for monitoring ARGs in multiple environmental samples ( $\mathrm{Li}$ et al., 2015b). Although the feasibility of ARG indicators has been tested in this study, we suggest that the accuracy should be more widely tried and tested in different environmental samples.

The co-occurrence patterns between ARGs and microbial taxa (Fig. 5), suggested that four bacterial families were thought to be possible ARGs hosts. Only few of the ARGs hosts have been verified in previous studies, for instance, by using network analysis, Li et al. found that Blautia was the host of tet32, tetM, tetQ and tetO and ermB, and Escherichia carry resistance genes of $c f x A 3$, tetQ acrA, $m d t H, m d t L$ and mdtO (Li et al., 2015b). Forslund et al. suggested that Blautia and Clostridium harbored tet32, tetO and ermB, whereas Escherichia was the host of $a c r A, m d t H, m d t L$ and $m d t O$ (Forslund et al., 2013). Network analysis is a reasonable tool to provide us new insights into the ARGs and their possible hosts in complex environmental samples (Ju et al., 2016). Nevertheless, the robustness of network analysis in predicting ARGs host need substantial further studies based on characterization of ARB and metagenomic survey of environmental samples.

\subsection{Responses of bacterial pathogens}

Two important human pathogens, Mycobacterium tuberculosis and Pseudomonas aeruginosa were detected. Previous studies have suggested that dominant human pathogens in manure were Bacillus anthracis and Bordetella pertussis (Fang et al., 2015), and those in sewage sludge were Escherichia coli and Klebsiella pneumoniae (Ye and Zhang, 2011), however, we did not detect these in our samples. One reasonable explanation is that these pathogens detected in sewage sludge or manures could not successfully establish in the present soil. This hypothesis is supported by a recent study, in which, it was reported that although Bacillus anthracis and Bordetella pertussis were detected in manure, they were not detected in manure-amended soil (Fang et al., 2015). In this study, the identification of bacterial pathogens was based on the use of the 16S rRNA gene sequence from each sample blast against the bacterial pathogen $16 \mathrm{~S}$ rRNA database. Although this is an effective way for pathogen detection, it still has the following limitations. Just like all the other PCR-based methods, the inherent amplification biases during PCR step could not be avoided (Haft and Tovchigrechko, 2012). Furthermore, the relative short sequence length (392 bp in this study) can only provide a taxonomic resolution at genus level (Segata et al., 2012), but not all of the species under the pathogenic genera are pathogens. These limitations also, at least in part, explain that we did not detect any significant difference in bacterial pathogens between the control and the sewage sludgetreated samples. Thus, we suggest that the metagenomic approach is a new methodology which could provide a broad and accurate profile of microbial communities and it should be used in bacterial pathogen identification in the future studies.

\section{Conclusions}

This study reveals that the long-term application of sewage sludge and chicken manure can increase the abundance and diversity of ARGs and bacteria. These results provide solid evidence for the correlation among antibiotic resistome, bacterial community and environment factors in the field, which may help better understand the promotion mechanisms of antibiotic resistance by soil organic amendments. To reduce the potential risk, we suggest that more efforts should be devoted to reducing the ARGs in sewage sludge and manure before land application. Additionally, network analysis could be a powerful tool to identify indicator ARGs in a complex interaction, for a quick estimation of ARGs in multiple environmental samples, and to delineate the possible ARGs hosts.

\section{Acknowledgments}

This work was supported by the National Natural Science Foundation of China (21210008, 41571130063) and the Strategic Priority Research Program of Chinese Academy of Sciences (XDB15020402).

\section{Appendix A. Supplementary data}

Supplementary data to this article can be found online at http://dx. doi.org/10.1016/j.envint.2016.03.026.

\section{References}

Binh, C.T.T., Heuer, H., Kaupenjohann, M., Smalla, K., 2008. Piggery manure used for soil fertilization is a reservoir for transferable antibiotic resistance plasmids. FEMS Microbiol. Ecol. 66, 25-37.

Binh, C.T.T., Heuer, H., Kaupenjohann, M., Smalla, K., 2009. Diverse aadA gene cassettes on class 1 integrons introduced into soil via spread manure. Res. Microbiol. 160, 427-433.

Bondarczuk, K., Markowicz, A., Piotrowska-Seget, Z., 2016. The urgent need for risk assessment on the antibiotic resistance spread via sewage sludge land application. Environ. Int. 87, 49-55. 
Burch, T.R., Sadowsky, M.J., LaPara, T.M., 2014. Fate of antibiotic resistance genes and class 1 integrons in soil microcosms following the application of treated residual municipal wastewater solids. Environ. Sci. Technol. 48, 5620-5627.

Candela, L., Fabregat, S., Josa, A., Suriol, J., Vigues, N., Mas, J., 2007. Assessment of soil and groundwater impacts by treated urban wastewater reuse. A case study: application in a golf course (Girona, Spain). Sci Total Environ. 374, 26-35.

Chen, H., Zhang, M.M., 2013. Occurrence and removal of antibiotic resistance genes in municipal wastewater and rural domestic sewage treatment systems in eastern China. Environ. Int. 55, 9-14.

Chen, Q.L., Wang, H., Yang, B.S., He, F., 2014. The combined effects of atrazine and lead $(\mathrm{Pb})$ : relative microbial activities and herbicide dissipation. Ecotoxicol. Environ. Saf. 102, 93-99.

Chen, Q.L., Wang, H., Yang, B.S., He, F., Han, X.M., Song, Z.H., 2015. Responses of soil ammonia-oxidizing microorganisms to repeated exposure of single-walled and multi-walled carbon nanotubes. Sci. Total Environ. 505, 649-657.

Cheng, W.X., Chen, H., Su, C., Yan, S.H., 2013. Abundance and persistence of antibiotic resistance genes in livestock farms: a comprehensive investigation in eastern China. Environ. Int. 61, 1-7.

Chopra, I., Roberts, M., 2001. Tetracycline antibiotics: mode of action, applications, molecular biology, and epidemiology of bacterial resistance. Microbiol. Mol. Biol. Rev. 65 (2), 232-260.

Edgar, R.C., 2010. Search and clustering orders of magnitude faster than BLAST. Bioinformatics 26 (19), 2460-2641.

Fahrenfeld, N., Knowlton, K., Krometis, L.A., Hession, W.C., Xia, K., Lipscomb, E., Libuit, K., Green, B.L., Pruden, A., 2014. Effect of manure application on abundance of antibiotic resistance genes and their attenuation rates in soil: field-scale mass balance approach. Environ. Sci. Technol. 48, 2643-2650.

Fang, H., Wang, H.F., Cai, L., Yu, Y.L., 2015. Prevalence of antibiotic resistance genes and bacterial pathogens in long-term manured greenhouse soils as revealed by metagenomic survey. Environ. Sci. Technol. 49, 1095-1104.

Forsberg, K.J., Reyes, A., Wang, B., Selleck, E.M., Sommer, M.O., Dantas, G., 2012. The shared antibiotic resistome of soil bacteria and human pathogens. Science 337, 1107-1111.

Forsberg, K.J., Patel, S., Gibson, M.K., Lauber, C.L., Knight, R., Fierer, N., Dantas, G., 2014. Bacterial phylogeny structures soil resistomes across habitats. Nature 509, 612-616.

Forslund, K., Sunagawa, S., Kultima, J.R., Mende, D.R., Arumugam, M., Typas, A., 2013. Country-specific antibiotic use practices impact the human gut resistome. Genome Res. 23, 1163-1169.

Gaze, W.H., Zhang, L.H., Abdouslam, N.A., Hawkey, P.M., Calvo-Bado, L., Royle, J., Brown, H., Davis, S., Kay, P., Boxall, A.B.A., Wellington, E.M.H., 2011. Impacts of anthropogenic activity on the ecology of class 1 integrons and integron-associated genes in the environment. ISME J. 5, 1253-1261.

Ghosh, S., LaPara, T.M., 2007. The effects of subtherapeutic antibiotic use in farm animals on the proliferation and persistence of antibiotic resistance among soil bacteria. ISME J. 1 (3), 191-203.

Gillings, M., Boucher, Y., Labbate, M., Holmes, A., Krishnan, S., Holley, M., Stokes, H.W., 2008. The evolution of class 1 integrons and the rise of antibiotic resistance. J. Bacteriol. 190, 5095-5100.

Haft, D.H., Tovchigrechko, A., 2012. High-speed microbial community profiling. Nat. Methods 9 (8), 793-794.

Halary, S., Leigh, J.W., Cheaib, B., Lopez, P., Bapteste, E., 2010. Network analyses structure genetic diversity in independent genetic worlds. Proc. Natl. Acad. Sci. U. S. A. 107, 127-132.

Hammesfahr, U., Heuer, H., Manzke, B., Smalla, K., Thiele-Bruhn, S., 2008. Impact of the antibiotic sulfadiazine and pig manure on the microbial community structure in agricultural soils. Soil Biol. Biochem. 40, 1583-1591.

Heuer, H., Focks, A., Lamshöft, M., Smalla, K., Matthies, M., Spiteller, M., 2008. Fate of sulfadiazine administered to pigs and its quantitative effect on the dynamics of bacterial resistance genes in manure and manured soil. Soil Biol. Biochem. 40, 1892-1900.

Jechalke, S., Kopmann, C., Rosendahl, I., Groeneweg, J., Weichelt, V., Krögerrecklenfort, E. Brandes, N., Nordwig, M., Ding, G.C., Siemens, J., Heuer, H., Smalla, K., 2013. Increased abundance and transferability of resistance genes after field application of manure from sulfadiazine-treated pigs. Appl. Environ. Microbiol. 79, 1704-1711.

Jia, S.Y., Shi, P., Hu, Q., Li, B., Zhang, T., Zhang, X.X., 2015. Bacterial community shift drives antibiotic resistance promotion during drinking water chlorination. Environ. Sci. Technol. 49, 12271-12279.

Jiang, X., Morgan, J., Doyle, M.P., 2002. Fate of Escherichia coli 0157:H7 in manureamended soil. Appl. Environ. Microbiol. 68, 2605-2609.

Joy, S.R., Bartelt-Hunt, S.L., Snow, D.D., Gilley, J.E., Woodbury, B.L., Parker, D.B., Marx, D.B., $\mathrm{Li}, \mathrm{X} ., 2013$. Fate and transport of antimicrobials and antimicrobial resistance genes in soil and runoff following land application of swine manure slurry. Environ. Sci. Technol. 47 (21), 12081-12088.

Ju, F., Li, B., Ma, L.P., Wang, Y.B., Huang, D.P., Zhang, T., 2016. Antibiotic resistance genes and human bacterial pathogens: co-occurrence, removal, and enrichment in municipal sewage sludge digesters. Water Res. 91, 1-10.

Kim, S., Aga, D.S., 2007. Potential ecological and human health impacts of antibiotics and antibiotic-resistant bacteria from wastewater treatment plants. J. Toxicol. Environ. Health-Part B-Crit. Rev. 10, 559-573.

Kyselková, M., Kotrbová, L., Bhumibhamon, G., Chroňáková, A., Jirout, J., Vrchotová, N., Schmitt, H., Elhottová, D., 2015. Tetracycline resistance genes persist in soil amended with cattle feces independently from chlortetracycline selection pressure. Soil Biol. Biochem. 81, 259-265.
Lachmayr, K.L., Kerkhof, L.J., Dirienzo, A.G., Cavanaugh, C.M., Ford, T.E., 2009. Quantifying nonspecific TEM $\beta$-lactamase (blaTEM) genes in a wastewater stream. Appl. Environ. Microbiol. 75, 203-211.

Li, B., Ju, F., Cai, L., Zhang, T., 2015a. Profile and fate of bacterial pathogens in sewage treatment plants revealed by high-throughput metagenomic approach. Environ. Sci. Technol. 49, 10492-10502.

Li, B., Yang, Y., Ma, L.P., Ju, F., Guo, F., Tiedje, J.M., Zhang, T., 2015b. Metagenomic and network analysis reveal wide distribution and co-occurrence of environmental antibiotic resistance genes. ISME J. 9, 2490-2502.

Littlefield-Wyer, J.G., Brooks, P., Katouli, M., 2008. Application of biochemical fingerprinting and fatty acid methyl ester profiling to assess the effect of thepesticide atradex on aquatic microbial communities. Environ. Pollut. 153, 393-400.

Munir, M., Wong, K., Xagoraraki, I., 2011. Release of antibiotic resistant bacteria and genes in the effluent and biosolids of five wastewater utilities in Michigan. Water Res. 45 681-693.

Ng, L.K., Martin, I., Alfa, M., Mulvey, M., 2011. Multiplex PCR for the detection of tetracycline resistant genes. Mol. Cell. Probes 15 (4), 209-215.

Ou-yang, W.Y., Huang, F.Y., Zhao, Y., Li, H., Su, J.Q., 2015. Increased levels of antibiotic resistance in urban stream of Jiulongiiang River. China Appl. Microbiol. Biotechnol. 99, 5697-5707.

Partridge, S.R., Tsafnat, G., Coiera, E., Iredell, J.R., 2009. Gene cassettes and cassette arrays in mobile resistance integrons. FEMS Microbiol. Rev. 33, 757-784.

Pruden, A., Pei, R.T., Storteboom, H., 2006. Antibiotic resistance genes as emerging contaminants: studies in northern Colorado. Environ. Sci. Technol. 40 (23), 7445-7450.

Rahube, T.O., Marti, R., Scott, A., Tien, Y.C., Murray, R., Sabourin, L., Zhang, Y., Duenk, P., Lapen, D.R., Topp, E., 2014. Impact of fertilizing with raw or anaerobically-digested sewage sludge on the abundance of antibiotic-resistant coliforms, antibiotic resistance genes and pathogenic bacteria in soil, and on vegetables at harvest. Appl. Environ. Microbiol. 80 (22), 6898-6907.

Schmittgen, T.D., Livak, K.J., 2008. Analyzing real-time PCR data by the comparative CT method. Nat. Protoc. 3 (6), 1101-1108.

Segata, N., Waldron, L., Ballarini, A., Narasimhan, V., Jousson, O., Huttenhower, C., 2012 Metagenomic microbial community profiling using unique clade-specific marker genes. Nat. Methods 9 (8), 811-814.

Smalla, K., Heuer, H., Götz, A., Niemeyer, D., Krögerrecklenfort, E., Tietze, E., 2000. Exogenous isolation of antibiotic resistance plasmids from piggery manureslurries reveals a high prevalence and diversity of IncQ-like plasmids. Appl. Environ. Microbiol. 66 4854-4862.

Su, J.Q., Wei, B., Xu, C.Y., Qiao, M., Zhu, Y.G., 2014. Functional metagenomic characterization of antibiotic resistance genes in agricultural soils from China. Environ. Int. 65, 9-15.

Su, J.Q. Bei Wei, B., Ou-Yang, W.Y., Huang, F.Y.,Zhao, Y., Xu, H.J., Zhu, Y.G., 2015. Antibiotic resistome and its association with bacterial communities during sewage sludge composting. Environ. Sci. Technol. 49, 7356-7363.

Tang, X.J., Lou, C.L., Wang, S.X., Lu, Y.H., Liu, M., Hashmi, M.Z., Liang, X.Q., Li, Z.P., Liao, Y.L., Qin, W.J., Fan, F., Xu, J.M., Brookes, P.C., 2015. Effects of long-term manure applications on the occurrence of antibiotics and antibiotic resistance genes (ARGs) in paddy soils: evidence from four field experiments in south of China. Soil Biol Biochem. 90, 179-187.

Threedeach, S., Chiemchaisri, W., Watanabe, T., Chiemchaisri, C., Honda, R., Yamamoto, K., 2012. Antibiotic resistance of Escherichia coli in leachates from municipal solid waste landfills: comparison between semi-aerobic and anaerobic operations. Bioresour. Technol. 113, 253-258.

Wang, L.L., Oda, Y., Grewal, S., Morrison, M., Michel, F.C., Yu, Z.T., 2011. Persistence of resistance to erythromycin and tetracycline in swine manure during simulated composting and lagoon treatments. Microb. Ecol. 63 (1), 32-40.

Wang, F.H., Qiao, M., Su, J.Q., Chen, Z., Zhou, X., Zhu, Y.G., 2014. High throughput profiling of antibiotic resistance genes in Urban Park soils with reclaimed water irrigation. Environ. Sci. Technol. 48, 9079-9085.

Wu, N., Qiao, M., Zhang, B., Cheng, W.D., Zhu, Y.G., 2010. Abundance and diversity of tetracycline resistance genes in soils adjacent to representative swine feedlots in China. Environ. Sci. Technol. 44, 6933-6939.

Xiong, W.G., Sun, Y.X., Ding, X.Y., Zhang, Y.M., Zhong, X.X., Liang, W.F., Zeng, Z.L., 2015. Responses of plasmid-mediated quinolone resistance genes and bacterial taxa to (fluoro)quinolones-containing manure in arable soil. Chemosphere 119, 473-478.

Yang, Y., Li, B., Zou, S.C., Fang, H.H.P., Zhang, T., 2014. Fate of antibiotic resistance genes in sewage treatment plant revealed by metagenomic approach. Water Res. 62, 97-106.

Ye, L., Zhang, T., 2011. Pathogenic bacteria in sewage treatment plants as revealed by 454 pyrosequencing. Environ. Sci. Technol. 45, 7173-7179.

Yin, H.Q., Niu, J.J., Ren, Y.H., Cong, J., Zhang, X.X., Fan, F.L., Xiao, Y.H., Zhang, X., Deng, J., Xie, M., He, Z.L., Zhou, J.Z., Liang, Y.L., Liu, X.D., 2015. An integrated insight into the response of sedimentary microbial communities to heavy metal contamination. Sci. Report. 5, 14266-14277.

Zhou, Z., Raskin, L., Zilles, J.L., 2010. Effects of swine manure on macrolide, lincosamide, and streptogramin B antimicrobial resistance in soils. Appl. Environ. Microbiol. 76 (7), 2218-2224.

Zhu, Y.G., Johnson, T.A., Su, J.Q., Qiao, M., Guo, G.X., Stedtfeld, R.D., Hashsham, S.A., Tiedje, J.M., 2013. Diverse and abundant antibiotic resistance genes in Chinese swine farms. Proc. Natl. Acad. Sci. U. S. A. 110, 3435-3440. 